\title{
Intracranial Atherosclerosis: From Microscopy to High-Resolution Magnetic Resonance Imaging
}

\author{
Wen-jie Yang, Ka-sing Wong, Xiang-yan Chen \\ Department of Medicine and Therapeutics, The Chinese University of Hong Kong, Prince of Wales Hospital, Sha Tin, Hong Kong
}

Intracranial atherosclerosis is one of the leading causes of ischemic stroke and occurs more commonly in patients of Asian, African or Hispanic origin than in Caucasians. Although the histopathology of intracranial atherosclerotic disease resembles extracranial atherosclerosis, there are some notable differences in the onset and severity of atherosclerosis. Current understanding of intracranial atherosclerotic disease has been advanced by the high-resolution magnetic resonance imaging (HRMRI), a novel emerging imaging technique that can directly visualize the vessel wall pathology. However, the pathological validation of HRMRI signal characteristics remains a key step to depict the plaque components and vulnerability in intracranial atherosclerotic lesions. The purpose of this review is to describe the histological features of intracranial atherosclerosis and to state current evidences regarding the validation of MR vessel wall imaging with histopathology.

Keywords Intracranial atherosclerosis; Autopsy; Histology; Magnetic resonance imaging

\author{
Correspondence: Xiangyan Chen \\ Department of Medicine and \\ Therapeutics, The Chinese University \\ of Hong Kong, Prince of Wales \\ Hospital, General Office, 9/F, Clinical \\ Sciences Building, Sha Tin, Hong Kong \\ Tel: +852-26352130 \\ Fax: $+852-26493761$ \\ E-mail: fionachen@cuhk.edu.hk \\ Received: December 8, 2016 \\ Revised: February 26, 2017 \\ Accepted: March 31, 2017 \\ The authors have no financial conflicts \\ of interest.
}

\section{Introduction}

Intracranial atherosclerosis (ICAS) is a major cause of stroke worldwide, accounting for $30-50 \%$ and $10 \%$ of ischemic cerebrovascular events in Asians and Whites, respectively. ${ }^{1-4}$ Over the last decades, there has been significant progress in the diagnosis and aggressive medical therapy of ICAS. Nevertheless, the histopathology of ICAS has not been well studied as extracranial atherosclerosis (ECAS), probably due to the infrequency of ICAS in Caucasians and the relative inaccessibility of intracranial arteries.

The only few large autopsy studies were performed during the 1960s and 1970s, exploring the distribution and natural history of large artery atherosclerosis in patients with all causes of death from fetuses to adults. They assessed the extent and severity of atherosclerosis in the aorta, coronary, carotid, and cerebral arteries, demonstrating that the cerebral arteries were free from atherosclerosis up to the fourth decade, almost 20 to 30 years later than extracranial arteries like coronary and carotid artery. ${ }^{5-7}$ Not only started later in life, ICAS was much slighter in the amount and extent than aorta and coronary artery atherosclerosis for all decades., ${ }^{5,8}$ The alterations observed in middle cerebral artery (MCA) in the 6th decade were similar to those in the radial artery in 4th decade and that in the coronary artery in 2-3th decades. ${ }^{9}$

In spite of the identification of less severe patterns of ICAS than ECAS in most autopsy studies, some racial differences were noted when comparing cerebral artery specimens from western and eastern populations. ${ }^{10,11}$ Compared to the late onset of intracranial atherosclerotic lesions in Caucasians, various degrees of ICAS were detected in patients with younger age in Asians. ${ }^{12-15}$ While Caucasians tend to have more ECAS, blacks and Asians tend to have more common and more severe patterns of ICAS..$^{16-19}$ A post-mortem study recruiting 114 Hong Kong Chinese autopsy cases demonstrated that the percent incidence of extreme atherosclerotic narrowing ( $>75 \%$ luminal 
narrowing) is much higher in the intracranial vessels than extracranial vessels. ${ }^{20}$ The prevalence and patterns of ICAS shown by autopsy studies are consistent with the data detected with different modalities in population-based or hospital-based studies demonstrating the predominant occurrence of ICAS in Asians and blacks. ${ }^{21}$ Unfortunately, the early macroscopic examination only described the prevalence and severity of ICAS by characterizing the surface involvement of fatty streak, plaque, ulcerated lesion, and calcification changes, failing to provide the detailed features of individual intracranial atherosclerotic lesions.

Currently, the conventional lumenography-based neuroimaging modalities, such as magnetic resonance angiography (MRA), computed tomography angiography (CTA), and digital subtraction angiography (DSA), are commonly used in the routine clinical practice to detect ICAS. However, evidences showed that luminal stenosis was insufficient in characterizing intracranial atherosclerotic lesions and accessing clinical risk. Till recently, high-resolution magnetic resonance imaging (HRMRI) emerges as a novel imaging modality to depict the pathologic changes in the vessel wall of minute arteries like intracranial arteries. ${ }^{22,23}$ The interpretation of signal intensity changes on HRMRI motivated researchers to explore the detailed histopathological elements within intracranial atherosclerotic lesions. Considering that intracranial artery specimens could not be acquired through clinical interventions as extracranial carotid specimens from carotid endarterectomy, collecting post-mortem cerebral vessel specimens is the most direct method to investigate the pathological characteristics of ICAS in human.

In this review, we will discuss the histopathological evidences of ICAS and its adoption in interpreting the signal changes detected on vessel wall imaging (VWI), which may benefit developing HRMRI to be a well-validated imaging technology for stroke patients.

\section{Histopathological evidence from human cadavers}

\section{Basic structure}

Atherosclerosis is a systemic and generalized disease involving intracranial and extracranial arteries. Patients with simple aortic plaques are frequently found to have concomitant ICAS, ${ }^{24,25}$ suggesting that patients with ICAS may share some common risk factors similar to those with aortic disease. Till now, our knowledge about the pathology of atherosclerosis is mostly acquired from studies on coronary artery disease, which illustrates that both luminal stenosis and plaque components indi- cating plaque vulnerability play an important role in leading to ischemic events. ${ }^{26}$ However, the correlation of coronary atherosclerosis with ICAS was found to be less close than that with extracranial carotid atherosclerosis, regardless of the classic risk factors for atherosclerosis. ${ }^{27}$ The different features of ICAS from ECAS may be attributable to the unique basic structures of intracranial cerebral arteries.

The basic organization of intracranial arteries is similar to extracranial arteries that consist three concentric layers: an inner layer (intima), a middle layer (media), and an outer layer (adventitia). But intracranial arteries exhibit some unique histological features that are quite different from those of comparatively sized vessels elsewhere in the body: a denser internal elastic lamina (IEL), a thinner media with slight development of elastic fibers, and a less abundant adventitia with decreased elastic fibers and without external elastic lamina (Figure 1). ${ }^{28}$ But how do the structure features peculiar to intracranial arteries account for the discrepancy in the formation and evolution of ICAS with ECAS? A previous post-mortem study compared the aging of MCA with that of other arteries of the same size, showing that the IEL was largely destroyed or even disappearing in the coronary artery while no marked splitting or dehiscence was observed in MCA. ${ }^{9}$ Though the exact mechanisms remain elusive, we speculate that the great prominence of the IEL may account for its less pronounced alterations in MCA, which, thus, protect the cerebral arteries from atheroma progressing outward. Although atherosclerosis is generally considered as a disease of the intima, the media and adventitia are also suggested to be related with the generation of the disease. Evidences showed that the smooth muscle cells within the media of different arterial beds have different embryonic origins, which may respond differently to the stimuli that facilitate the formation of atherosclerotic lesions. Besides, the matrix molecules surrounding smooth muscle cells, such as fibrillar collagen, may also considerably affect the inflammatory and fibrop-

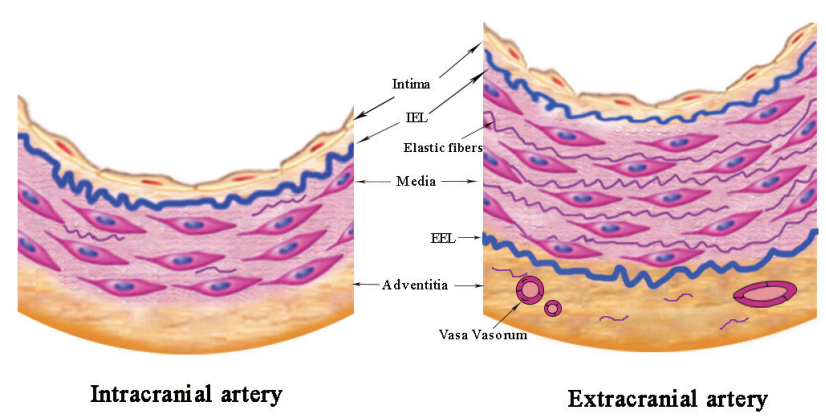

Figure 1. Basic structure of intracranial and extracranial arteries. IEL, internal elastic lamina; EEL, external elastic lamina. 
roliferative response, thus influencing the progression of atherosclerosis. ${ }^{29}$ Given that the intracranial arteries, different from the extracranial arteries being elastic arteries, are muscular arteries with little elastic fibers, the different embryonic derivation of smooth muscle cells and distinct composition of matrix within the intracranial arteries may explain the relative benign nature of ICAS.

In addition, some researchers argue that the thinner media and adventitia as well as the lack of external elastic lamina may be responsible for the relative paucity of vasa vasorum $(\mathrm{V})$ in intracranial arteries, ${ }^{30,31}$ which would further influence the formation of ICAS. W constitute a network of microvessels that supply the vessel wall oxygen and nourishment, and eliminate the wastes. Unlike the extracranial W existing at birth, intracranial $\mathrm{W}$ develop later in adulthood, as a response to tissue hypoxia when the vessels thicken and become diseased. ${ }^{32,33}$ The existence of $\mathrm{W}$ in human intracranial arteries has long been debated. Current evidences suggested that $\mathrm{W}$ were predominantly found in the proximal parts of the intracranial arteries, such as the distal ICA, vertebral arteries (VA), basilar arteries (BA), and MCA M1 segments, ${ }^{33,34}$ which may represent an extension of the vascular pathologies from the extracranial to the intracranial segments of cerebral arteries. One reason that may explain the rarity of intracranial $W$ is that the intracranial arteries are surrounded by nutrient-rich cerebro-spinal fluid (CSF), which may reduce the needs for nutrient and oxygen supply through W. Besides, as stated above, the intracranial arteries possess certain features different from similar sized extracranial vessels, namely, a relative paucity of elastic tissue in both the thinner media and less abundant adventitia, and lack of external elastic lamina. These features may facilitate the diffusion of oxygen and nutrition from the CSF, making the intracranial $\mathrm{W}$ less necessary.

During the past few years, several studies have been performed on experimental animals and human autopsy cases, indicating that $\mathrm{W}$ may play a critical role in the atherosclerotic plaque initiation, progression and destabilization. ${ }^{30,35,36}$ Evidences suggested that $\mathrm{W}$ may serve as a conduit in the inflammatory process of atherosclerosis, transporting the inflammatory cells into the plaque, which promoted the development of atherosclerosis. ${ }^{37,38}$ Besides, most of the $\mathrm{W}$ that grow into the plaque are immature with week integrity, resulting in the leakage of blood constituents and subsequent plaque hemorrhage. ${ }^{39}$ Therefore, $\mathrm{W}$ were considered as an independent predictor of atherosclerotic plaque vulnerability and associated with the symptomatic ischemic stroke. However, whether $\mathrm{W}$ in intracranial arteries play a causative role in the atherosclerotic process is not yet well established. Yet, the presence of $\mathrm{W}$ has been suspected as a specific marker in the early detection of vulnerable plaques and a new surrogate target in the atherosclerotic prevention and treatment. ${ }^{40}$ Given the critical role of $W$ in the process of atherosclerosis, the relative paucity of intracranial $\mathrm{W}$ may explain the late onset and relatively benign features of ICAS.

The size of vessels is a basic parameter to develop new imaging modalities to "visualize" the vessels in vivo. The quantitative measurements of extracranial carotid and coronary artery size have been made on autopsy or by angiographic or sonographic techniques. ${ }^{41,42}$ The left main artery, which is the largest major division of coronary artery, is about $5 \mathrm{~mm}$ in diameter, while the other major branches are approximately $3 \mathrm{~mm}^{43}$ The internal carotid artery is about $5 \mathrm{~mm}$ above the carotid bulb and the common carotid artery is more than $6 \mathrm{~mm}$ at about 15 to $20 \mathrm{~mm}$ below the bifurcation in diameter. ${ }^{44} \mathrm{In}$ contrary, compared with the extensively studied extracranial arteries, the size of intracranial arteries has been rarely reported. To provide reference values for imaging studies involving intracranial arteries, we quantitatively measured 96 intracranial large arteries (including $32 \mathrm{MCAs}, 32 \mathrm{VAs}$, and $32 \mathrm{BAs}$ ) at the most stenotic sites, which were collected from 32 consecutively recruited Chinese general autopsy cases (mean age: 71 years; male: $72 \%$; causes of death: coronary artery disease, $n=13$ [41\%]; infection or sepsis, $n=3$ [9\%]; other natural causes, $n=13$ [41\%]; unnatural causes, $n=3$ [9\%]) (Supplementary Methods 1, Supplementary Table 1). Table 1 shows the reference values of vessel diameters and vessel wall thickness for intracranial large arteries. The arterial sizes were $3.13 \pm 0.52$ $\mathrm{mm}, 3.46 \pm 0.73 \mathrm{~mm}$, and $3.60 \pm 0.66 \mathrm{~mm}$ in diameters for MCA, $V A$, and BA, respectively, consistent with a previous autopsy study (proximal internal carotid artery $=3.9 \mathrm{~mm}$, proximal $M C A=3.1 \mathrm{~mm}$, proximal $V A=3.2 \mathrm{~mm}$, proximal $B A=3.7 \mathrm{~mm}$, respectively). ${ }^{45}$ The normal media of MCA, VA, and BA ranged in thickness from $0.15 \mathrm{~mm}$ to $0.19 \mathrm{~mm}$, a slightly thinner than that of coronary arteries $(0.13-0.35 \mathrm{~mm})$. The medial thickness behind the atherosclerotic plaque were much lower, being $0.10 \pm 0.05 \mathrm{~mm}, 0.11 \pm 0.06 \mathrm{~mm}$, and $0.12 \pm 0.07 \mathrm{~mm}$ for MCA, $V A$, and $B A$, respectively. The adventitial thickness ranged from $0.05 \mathrm{~mm}$ to $0.10 \mathrm{~mm}$, considerably thinner than that of coronary arteries $(0.3-0.5 \mathrm{~mm}){ }^{46}$ The media thickness compared to arterial radius was similar among MCA, VA, and BA (9.5\%, $11.3 \%, 11.5 \%$, respectively), but VA had a thicker adventitia in comparison with MCA (6.2\% vs. $3.2 \%, P=0.001)$ and BA (6.2\% vs. $3.9 \%, P=0.008$ ) (Figure 2). In our recent study, we found that VA had more W than BA and MCA (submitted) (Figure 2), which may be attributed to the thicker adventitia percentage in VA. Based on the collected intracranial vessels, the severity 
Table 1. Intracranial arterial characteristics by arterial segments

\begin{tabular}{lccc}
\hline & $\begin{array}{c}\text { Middle cerebral artery } \\
(\mathrm{n}=32)\end{array}$ & Vertebral artery $(\mathrm{n}=32)$ & Basilar artery $(\mathrm{n}=32)$ \\
\hline Arterial diameter $(\mathrm{mm})$ & $3.13 \pm 0.52$ & $3.46 \pm 0.73$ & $3.60 \pm 0.66$ \\
Media thickness $(\mathrm{mm})$ & $0.15 \pm 0.05$ & $0.19 \pm 0.10$ & $0.19 \pm 0.07$ \\
Media behind plaque $(\mathrm{mm})$ & $0.10 \pm 0.05$ & $0.11 \pm 0.06$ & $0.12 \pm 0.07$ \\
Adventitia thickness $(\mathrm{mm})$ & $0.05 \pm 0.03$ & $0.10 \pm 0.06$ & $0.06 \pm 0.02$ \\
Adventitia behind plaque $(\mathrm{mm})$ & $0.05 \pm 0.03$ & $0.11 \pm 0.07$ & $0.05 \pm 0.05$ \\
Media ratio to arterial radius $(\%)$ & $9.5 \pm 3.5$ & $11.1 \pm 4.9$ & $11.6 \pm 3.7$ \\
Adventitia ratio to arterial radius (\%) & $3.2 \pm 1.8$ & $5.8 \pm 3.2$ & $3.9 \pm 1.7$ \\
Area stenosis $(\%)$ & $37 \pm 25$ & $30 \pm 24$ & $20 \pm 20$ \\
\hline
\end{tabular}


Figure 2. Large intracranial arterial segments with hematoxylin- eosin staining. (A) Middle cerebral artery. (B) Basilar artery. (C) Vertebral artery with vasa vasorum. Original magnification: $1.6 \times 1.6$.

of luminal stenosis was significantly different, showing $37 \pm 25 \%$ at MCAs, $30 \pm 24 \%$ at VAs, and $20 \pm 20 \%$ at BAs (all $P<0.05)$, respectively.

\section{Histopathological features of ICAS}

Atherosclerotic plaques consist of cells, connective tissue extracellular matrix, and intra- and extra-cellular lipid deposits. The proportion of these three components varies in different atherosclerotic plaques, and it affects plaques stability and gives rise to a wide spectrum of lesions: 1) The so-called fatty streaks are characterized by adhesion of monocytes to the endothelium and migration to the subendothelial potions of the arterial wall in the adolescents and young adults; 2) With increasing age, fatty streaks are transformed into fibrous plaques consisting of a core of cellular debris, free extracellular lipid, and cholesterol crystals under a "cap" of foam cells, transformed smooth muscle cells, lymphocytes, and connective tissue; and 3) The most advanced stage of atherosclerosis is the complicated lesion, which includes calcification, hemosiderin deposition, and lumen surface disruption. ${ }^{47,48}$

Atherosclerosis occurs commonly at the early age or even soon after birth, but majority of them keep as a stable state, which will not cause any clinical events in the whole life, while only those at high risk of precipitating life-threatening thrombi are responsible for leading to the eventual vascular diseases. For clinicians and researchers, the challenge is to enable identification of lesions that are vulnerable to thrombosis, the socalled "vulnerable plaques." Based on coronary artery atherosclerosis, the major criteria to define a vulnerable plaque are: 1) active inflammation, 2) a thin cap with a large lipid core, 3) endothelial denudation with superficial platelet aggregation, 4) fissured/injured plaque, and 5) severe stenosis; and the minor criteria include the following features: 1) superficial calcified nodules, 2) yellow color (on angioscopy), 3) intraplaque hemorrhage, 4) endothelial dysfunction, and 5) expansive (positive) remodeling. ${ }^{26,49}$ These criteria may be helpful to define vulnerable atherosclerotic plaques within intracranial vasculature.

Current evidences suggested that ICAS developed mainly as early lesions with more fibrosis in contradistinction to the eroded and ruptured plaques more commonly encountered in ECAS $^{50}$ The more advanced atherosclerotic changes were infrequently detected in intracranial artery until the very old 
years, ${ }^{16,51}$ such as calcified lesion first detected in the 6th decade, and ulcerated lesion first observed in the 9th decade. ${ }^{8} \mathrm{~A}$ study of 1,220 segments of circle of Willis from 67 randomly chosen autopsy cases showed that early plaques were predominant $(66 \%)$ and advanced plaques exceptional $(15 \%)$, whereas complicated plaques were present in only $1 \%$ of lesions. ${ }^{52}$ Figure 3 shows the plaque components on histology.

Intraplaque hemorrhage is a frequently detected marker in advanced atherosclerosis. In carotid artery involved with atherosclerosis, intraplaque hemorrhage was observed in as high as $19 \%$ to $97 \%$ within symptomatic lesions and $7 \%$ to $91 \%$ within asymptomatic lesions. ${ }^{53,54}$ By contrast, a much lower prevalence of intraplaque hemorrhage was reported in studies involving intracranial arteries: only one lesion with intraplaque hemorrhage was reported in 205 intracranial arterial segments ${ }^{55}$ and 6 of 1,220 segments had intraplaque hemorrhage among patients who had cardiovascular-related events. ${ }^{52}$ But the samples of these two studies were taken at specific locations per circle of Willis specimen, which may lead to an underestimate of the prevalence of intraplaque hemorrhage. Our previous study based on 76 Chinese autopsy adults demonstrated that intraplaque hemorrhage was much more prevalent in culprit plaques $(30 \%, 14 / 46)$ than in non-culprit plaques $(15 \%, 10 / 65) .{ }^{56}$ The incidence of intraplaque hemorrhage was much higher in our study than the western studies, but still lower than that in most extracranial artery studies. Recently, a HRMRI study recruiting 107 adult patients with high-grade (70\%) MCA stenosis also revealed a significantly higher prevalence of MRI-defined intraplaque hemorrhage in symptomatic lesions than that in asymptomatic lesions (19.6\% vs. 3.2\%, $P=0.01){ }_{1}^{57}$ consistent with our autopsy study. But the definition of intraplaque hemorrhage used in this study was extrapolated from carotid HRMRI-patholgy comparative studies, and a thorough histologic validation has not yet been performed in intracranial arteries.

Calcification is another frequently detected plaque component, which also indicates complexed or advanced atherosclerotic lesions. ${ }^{58,59}$ Our most recent retrospective study illustrated the correlation of high extent of internal carotid artery calcification with downstream microembolic signal detected by transcranial Doppler, suggesting that severe cerebral artery calcification might indicate complex or unstable atherosclerotic lesions. ${ }^{60}$ On histology, an autopsy study among Caucasians detected only $3 \%$ of intracranial arteries containing calcification, mostly detected in the VA, which was much less frequent than that in coronary and carotid arteries. ${ }^{52}$ Comparatively, we reported a high prevalence of calcification within cerebral arteries specimens from a series of Chinese adult autopsy cases: $35 \%(16 / 46)$ calcification within symptomatic MCA plaques and 23\% (15/65) within asymptomatic MCA plaques. ${ }^{56}$ Based on brain computed tomography (CT) results, we reported $60 \%$ of calcification at the internal carotid arteries, 20\% at VA, 5\% at BA and MCA in Chinese patients. ${ }^{61}$ The discrepancy in prevalence of calcification between histological evidence and CT detections could be due to the different criteria of defining calcification in pathology versus brain CT scans. The calcified nodules, a separate precipitating factor of thrombosis in rare cases, while present in approximately 6-7\% of carotid disease and $1-2 \%$ coronary artery disease ${ }_{1}^{62}$ has never been reported in the intracranial arteries.

In addition to intraplaque hemorrhage and calcification, inflammatory cell infiltration, especially macrophage accumulation, is a major player in inducing plaque activity. Special staining targeting macrophage or lymphocyte is a direct and acute method to evaluate inflammation involvement within atherosclerotic plaques. Within 283 circles of Willis segments from 18 asymptomatic elderly individuals, Ritz et al. reported a relatively lower frequency of macrophage load $(0.9 \pm 0.7 \%$ CD68 positivity per plaque area) compared with $1.8 \pm 2.4 \%$ within coronary arteries. ${ }^{28}$ Another pathological study of 8 stroke patients found mild to moderate inflammation in 3/7 BA atherosclerotic lesions. ${ }^{63}$ Histological data based on Chinese adult autopsy cases demonstrated more infiltrations of both macrophages (stained by CD68) and lymphocytes (stained by CD45RO) within atherosclerotic plaques associated with infarct than those within plaques not associated with infarct. ${ }^{56}$
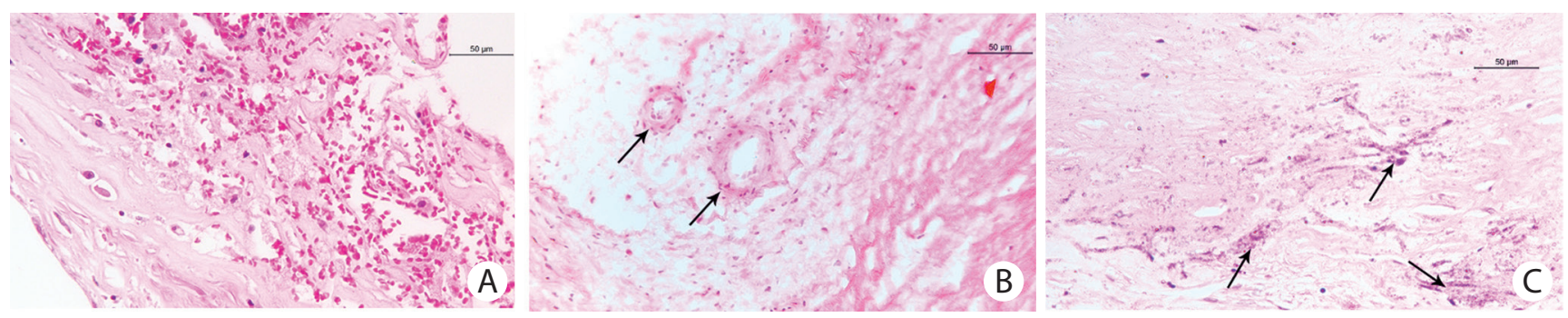

Figure 3. Plaque components on histology. (A) Intraplaque hemorrhage. (B) Neovascularue (arrows). (C) Calcification (arrows). Original magnification: $\times 20$. 
Beyond the plaque components mentioned above, other characteristics of a vulnerable plaque defined in coronary artery atherosclerosis, such as a thin fibrous cap, have not been investigated in the histology of ICAS. Although the current evidences seem to indicate that intracranial atherosclerotic plaques exhibit a relatively benign histopathological profile with rare neovasculature, infrequent inflammation, and rare ulceration and rupture ${ }_{1}^{63}$ further studies are required to explore the pathological features of intracranial atherosclerotic lesions, especially among Asian populations with a predominant prevalence of ICAS. The clarification of underlying pathophysiological mechanisms related to the progression of ICAS may be helpful to identify new imaging biomarkers for risk stratifying patients with ICAS and to provide targeted therapy aiming to stabilize the vulnerable atherosclerotic plaques.

\section{ICAS and ischemic stroke}

During the past few years, luminal stenosis of atherosclerotic vessels, which directly leads to insufficient blood supplying at downstream vessel segments, was regarded as a major indicator evaluating the severity of ICAS. However, recent studies demonstrated that the ipsilateral stroke rate was relatively low even in patients with severe stenosis, indicating that measuring the stenosis at the narrowest vessel segments may not be the optimal way to evaluate the clinical risk of ICAS.22 Many other features that reflect the characteristics of ICAS, such as plaque morphology and components, might be more precise in risk stratification of patients with ICAS.22,56,57,64

In 2008, a case-control autopsy study of 339 stroke patients first proposed that stenosis graded 30\% to $75 \%$ also played a causal role in the fatal stroke ${ }_{1}^{65}$ indicating that the definition based solely on stenosis could not fully capture all the risk of vascular events. Consistent with this, another study demonstrated that a substantial minority of cases showed advanced atherosclerotic phenotype while the degree of stenosis did not exceed $40 \% .{ }^{66}$ On the contrary, although severe atheromatous narrowing was common in intracranial arteries, the lesions were usually covered by an intact fibromuscular cap that was less prone to rupture, and ulceration and fissuring were rarely detected. ${ }^{20}$ The discrepancy in severity of stenosis versus histopathological phenotypes further supported the notion that the degree of stenosis does not fully account for cerebral atherosclerosis burden, highlighting the need to pay more attention to the plaque components and phenotype beyond the maximal luminal stenosis. Previously, we explored the clinical implications of plaque morphology and compositions in 76 consecutive Chinese postmortem adults, demonstrating that in addition to the luminal stenosis, plaque components such as lipid, intraplaque hemorrhage, neovasculature, and thrombus, may be responsible for the brain infarct. ${ }^{56}$ Therefore, besides the atherosclerosis-induced luminal stenosis, plaque components that indicate vulnerable plaques may also play a key role in leading to ischemic stroke. Imaging modalities that can identify these potentially high-risk plaque components will benefit in improving risk stratification of patients with ICAS and making treatment decision for individual patients.

\section{Histopathological validation of MR ves- sel wall imaging}

Over the past decades, luminal stenosis identified by the conventional imaging techniques remains the principal means for determining treatment decisions in patients with intracranial stenotic diseases. However, the findings from the Warfarin versus Aspirin for Symptomatic Intracranial Disease (WASID) trial ${ }^{67}$ and the Chinese Intracranial Atherosclerosis (CICAS) study ${ }^{68}$ demonstrated that, although the risk of stroke was highest with severe stenosis $(\geq 70 \%)$, a certain percentage of patients with moderate luminal stenosis (50-69\%) also suffered from the subsequent or recurrent ischemic stroke, implying that the luminal information is not sufficient in assessing the severity of ICAS and predicting the future cerebrovascular events. Therefore, it is urgently needed to develop a novel imaging method that could provide more information about the changes of vessel wall lesions, such as the plaque morphology and components within intracranial atherosclerotic lesions.

HRMRI technique is a promising tool which provides sufficient high spatial resolution to visualize the arterial lesions within much smaller sized intracranial vessels..$^{69,70}$ Moreover, this new VWI is capable of identifying intracranial arterial lesions even when luminal angiography reveals no abnormalities. $^{71}$ In recent years, the feasibility of HRMRI in quantitatively measuring intracranial arterial lesions has been demonstrated in several clinical studies. ${ }^{72-74}$ The capability of VWI in depicting the pathologic changes in intracranial vessels could also be used to differentiate various vascular pathologies other than atherosclerosis, such as dissection, moyamoya disease, vasculitis, and so on. ${ }^{75}$

In the area of extracranial large arteries such as carotid and coronary arteries, collecting arterial specimens from carotid endarterectomy provides a direct method to validate the capability of HRMRI in identifying carotid plaque components..$^{76-78}$ Currently, several studies have demonstrated the accuracy of HRMRI in assessing fibrous cap, lipid-rich necrotic core, or intraplaque hemorrhage by referring to pathological findings of specimens. ${ }^{79}$ However, it is risky to extrapolate these experiences from carotid 
HRMRI directly to intracranial arteries, because intracranial arteries are smaller in size and have distinct basic structures compared with extracranial vessels. ${ }^{56,63}$ Although a few recent clinical studies have reported some HRMRI-defined plaque components of ICAS, such as intraplaque hemorrhage, ${ }^{57}$ the signal interpretation on intracranial VWI is still a big hurdle due to the lack of dedicated histological validation.

Based on a biobank including intracranial arterial specimens from a series of Chinese adult autopsy cases, we investigated the signal changes on 1.5T VWI by comparing with the plaque components on histology. Consistent with the imaging features of $E C A S_{1}{ }^{57}$ the high signal on T1 sequence in ICAS was verified to be intraplaque hemorrhage on histology in an 83-year-old woman (Figure 4A, B). ${ }^{80}$

On the basis of the ex vivo 1.5T MR VWI-histology comparative study, we further illustrated that the low signal on T1weighted fat-suppressed images could be a promising imaging marker for identifying the intraplaque lipid core, and it showed a much higher sensitivity for detecting lipid core of area $\geq 0.8 \mathrm{~mm}^{2}$ (Figure 4C, D). ${ }^{81}$ Although the signal to noise ratio provided by $1.5 \mathrm{~T}$ MRI was not sufficient enough to confirm the findings in every autopsy case, the results based on the above comparative studies provided limited but valuable preliminary data to indicate the capability of MRI in characterizing the intracranial plaque components. Recently, another ex vivo study showed that the multi-contrast 3T MRI could clearly distinguish lipid core from the overlying fibrous cap by using T2- and T2*-weighted imaging, making it feasible to accurately identify the vulnerable fibro-lipid atheroma from the low risk plaque types, such as intimal thickening or fibrotic plaque without lipid core. ${ }^{82}$ Lipid core is an important plaque component, which may influence the plaque vulnerability. The identification of lipid core on VWI may provide valuable imaging basis for aggressive therapy in patients with ICAS.

Compared with $1.5 \mathrm{~T}$ or $3 \mathrm{~T}$ MRI, $7 \mathrm{~T}$ ultra-high resolution MR imaging that provides the higher spatial resolution necessary to image small intracranial vessel lesions may work better in distinguishing different plaque components. An ex vivo HRMRIhistology comparative study assessed the feasibility of 7T MR imaging to characterize intracranial atherosclerotic lesions using a single sequence with the mixed contrast of $\mathrm{T} 1$ and $\mathrm{T} 2$ weighting, first demonstrating that $7 \mathrm{~T}$ MRI has sufficient image contrast to enable differentiation of fibrosis and attenuated calcium deposition within the plaques. ${ }^{83}$ Another study collecting 44 arterial samples from five specimens of the circle of Willis also demonstrated the ability of 7T HRMRI to distinguish different types of plaque components, such as foamy macrophages and collagen, within more advanced plaques. ${ }^{84}$ By using 7T MRI with multiple contrast weightings, the same team further identified the MR signal features in a quantitative manner
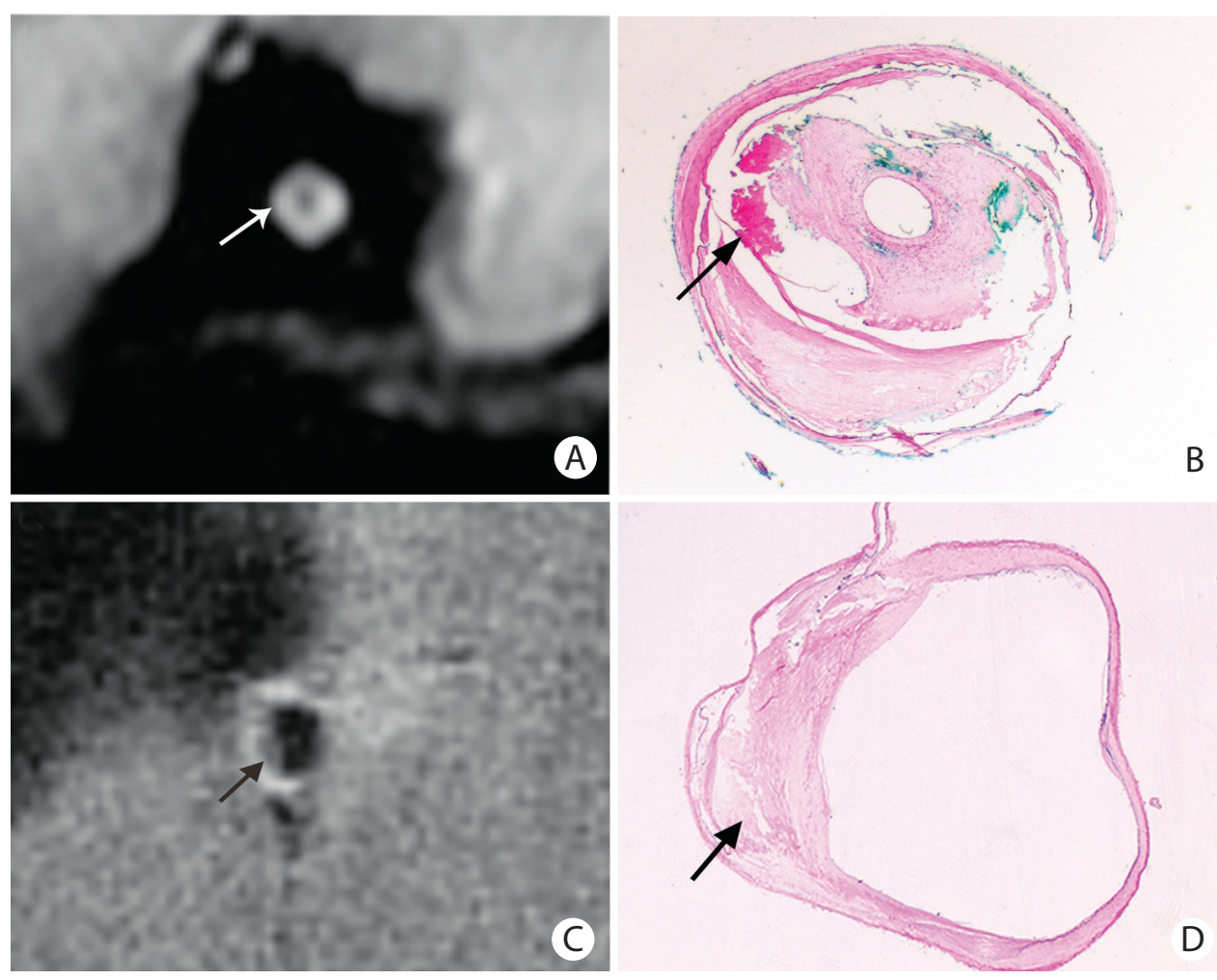

Figure 4. Representative magnetic resonance (MR) images and the corresponding histology slides. (A, B) The hyper-intense on MR images (arrow in A) was verified to be intraplaque hemorrhage (arrow in B). (C, D) The hypo-intense on MR images (arrow in $\mathrm{C}$ ) was verified to be lipid accumulation (arrow in D). The histological slides were stained with hematoxylin-eosin staining. Original magnification: $\times 1.6$. 
and showed that lipid accumulation, fibrous tissue, fibrous cap, and calcification within advanced intracranial atherosclerotic lesions had distinguishable imaging characteristics, especially on T1-weighted imaging. ${ }^{55}$ However, their findings on signal changes indicating some plaque components (e.g., lipid-rich core) were inconsistent with the signal characteristics validated in carotid artery studies, possibly due to the much earlier stage of intracranial atherosclerotic lesions or the prolonged formalin fixation of arterial specimens. However, despite the excellent resolution and signal-to-noise ratio, 7T MRI is less used in clinical practice due to the limited availability, high cost, and long scan duration.

Table 2 summarizes the plaque characteristics on VWI verified by histology. According to the current findings, intraplaque hemorrhage and large lipid core may be differentiated by VWI, especially on the fat-suppressed T1-weighted sequence. Fibrous cap shows more ambiguous signal intensity, perhaps because of the complicated tissue compositions or the insufficient resolution of VWI. Some studies reported the presence of a T2 juxtaluminal hyper-intense band overlying T2 hypo-intense components in intracranial atherosclerotic plaques, which was assumed to be corresponding to the fibrous cap based on the experiences in carotid atherosclerotic lesions, ${ }^{85,86}$ but the histologic validation has not yet been performed in ICAS. While some studies showed that calcified deposit in ICAS could be identified by using VWI, both the sensitivity and specificity were much lower when compared with CT scans. Strong contrast enhancement, which suggests a rich vascular supply into the plaque and incremental endothelial permeability, has been extensively studied in extracranial arteries. Validated by histology, several carotid studies demonstrated that gadolinium preferentially enhanced the fibrous cap and regions with extensive neovasculature and macrophages. ${ }^{87-89}$ Therefore, the carotid plaque enhancement seems to be a vital indicator of plaque vulnerability, which may assist in the stroke risk stratifi- cation. The same patterns of contrast enhancement were also observed in intracranial arteries, ${ }^{90-93}$ but the plaque compositions behind the enhancement have not yet been validated. More HRMRI-histology comparative studies are needed to interpret the imaging features in intracranial atherosclerotic lesions and thereby provide us more precise information about future risk related to ICAS.

Although the initial findings were promising, additional challenges remain. Firstly, despite the findings acquired from ex vivo $\mathrm{WWI}$-histology comparative studies, signal changes on MRI should be interpreted with cautions when applying the knowledge to in vivo studies. Compared with in vivo arteries filled with blood flow, ex vivo specimens had some degree of shrinkage after being fixed with formalin, which may change the signal intensity of arterial tissues on MRI. Secondly, although a comparison between in vivo MRI scanning and arterial specimens is the best way to validate the signal characteristics on $\mathrm{MRI}{ }^{94}$ the pathological specimens are very difficult to acquire in current interventional treatment. Finally, in spite of the high resolution of $\mathrm{VWI}$, it is still challengeable to characterize the intracranial atherosclerotic plaques, especially in the small vessels like anterior cerebral arteries. Therefore, there is a long way to go before HRMRI could be used as a routine imaging technique to assess intracranial plaque characteristics indicative of plaque vulnerability.

\section{Conclusions and future directions}

Compared with ECAS, ICAS develops later in life and shows more stable features, such as more early lesions with intimal thickening, infrequent intraplaque hemorrhage, and rare neovasculature and inflammation. But the earlier occurrence and much more severe patterns of intracranial atherosclerotic lesions were found in Asian population. The distinct characteristics of ICAS may be caused by unique anatomical structures of

Table 2. Plaque characteristics of intracranial atherosclerotic lesions on histology-verified vessel wall imaging (VWI)

\begin{tabular}{|c|c|c|}
\hline Plaque characteristics & Signal on the histology-validated VWI & References \\
\hline \multirow[t]{2}{*}{ Fibrous cap } & Iso-intense on $\mathrm{T} 1$ and $\mathrm{T} 2$ & Turan et al..$^{94}$ \\
\hline & Hyper-intense on T1 and T2 mixed sequence & Majidi et al..$^{83}$ \\
\hline Intraplaque hemorrhage & Hyper-intense on fat-suppressed T1 & Chen et al. ${ }^{80}$ \\
\hline \multirow[t]{3}{*}{ Lipid core } & Hypo-intense on fat-suppressed T1 & Yang et al. ${ }^{81}$ \\
\hline & Hyper-intense on T1 & Turan et al..$^{94}$ \\
\hline & Hypo-intense on T2 and T2* & Jiang et al. ${ }^{82}$, van der Kolk et al. ${ }^{84}$ \\
\hline Calcification & Hypo-intense on $\mathrm{T} 1$ and $\mathrm{T} 2$ & Turan et al..$^{94}$ \\
\hline Neovasculature & Contrast enhancement? & \\
\hline Inflammation & Contrast enhancement? & \\
\hline
\end{tabular}


intracranial arteries. Better understanding of the basic structures of intracranial arteries may help to elucidate the underlying mechanisms accounting for the natural history of ICAS.

Pathological findings from postmortem intracranial artery specimens demonstrated that, in addition to luminal stenosis caused by atherosclerotic lesions, the plaque burden and morphology as well as compositions were associated with brain infarcts. HRMRI emerges as a novel imaging technology to depict the vessel wall changes within intracranial arteries, holding the potential to quantitatively characterize the different tissue components. In extracranial carotid arteries, several studies have demonstrated the capacity of HRMRI to quantitatively characterize the morphological features related with the unstable plaques, such as intraplaque hemorrhage, necrotic core, and unstable fibrous cap. Recently, few MRI-histology comparative studies also demonstrated the feasibility of VWI in identifying the potentially high-risk plaque components (e.g., intraplaque hemorrhage, lipid core) in intracranial atherosclerotic lesions, opening a new exciting era in intracranial HRMR imaging. However, the inconsistency of the present results and the paucity of verifying some specific imaging characteristics like plaque enhancement call for further studies to fill a gap in evaluating the plaque activity by using intracranial HRMRI. With the advances of this MR technique, we hope it will have the ability to identify vulnerable plaques by using a variety of contrast enhancement techniques, just like in extracranial atherosclerotic plaques, which could ultimately permit the identification of "high-risk" patients with potential cerebrovascular complications. Also, the application of intracranial HRMRI in clinical practice may allow evaluate the predictive values of vulnerable plaque features in the subsequent ischemic events, and identify other imaging predictors for recurrent ischemic stroke. Finally, we hope that the intracranial HRMRI may direct the medical therapy and better evaluate their efficacies in patients with ICAS.

\section{Supplementary materials}

Supplementary materials related to this article can be found online at https://doi.org/10.5853/jos.2016.01956.

\section{References}

1. Kim JS, Bonovich D. Research on intracranial atherosclerosis from the east and west: why are the results different? J Stroke 2014;16:105-113.

2. Qureshi $A l$, Feldmann $E_{\text {, Gomez }} C R$, Johnston $S C$, Kasner $\mathrm{SE}_{1}$ Quick DC, et al. Intracranial atherosclerotic disease: an up- date. Ann Neurol 2009;66:730-738.

3. Komotar RJ, Kellner CP, Raper DM, Strozyk D, Higashida RT, Meyers PM. Update on the natural history of intracranial atherosclerotic disease: a critical review. World J Radiol 2010;2:166-171.

4. Suri MF, Johnston SC. Epidemiology of intracranial stenosis. J Neuroimaging 2009;19 Suppl 1:11S-16S.

5. Mathur KS, Patney NL, Kumar V. Atherosclerosis in india. An autopsy study of the aorta and the coronary, cerebral, renal, and pulmonary arteries. Circulation 1961;24:68-75.

6. Mathur KS, Kashyap SK, Kumar V. Correlation of the extent and severity of atherosclerosis in the coronary and cerebral arteries. Circulation 1963;27:929-934.

7. Baker $A B$, lannone A. Cerebrovascular disease. I. The large arteries of the circle of willis. Neurology 1959;9:321-332.

8. Sadoshima S, Kurozumi T, Tanaka K, Ueda K, Takeshita M, Hirota $Y$, et al. Cerebral and aortic atherosclerosis in Hisayama, Japan. Atherosclerosis 1980;36:117-126.

9. Bouissou H, Emery MC, Sorbara R. Age related changes of the middle cerebral artery and a comparison with the radial and coronary artery. Angiology 1975;26:257-268.

10. Gorelick PB. Distribution of atherosclerotic cerebrovascular lesions. Effects of age, race, and sex. Stroke 1993;24(12 Suppl 1):I16-I19; discussion I20-I21.

11. Kim JS, Kim YJ, Ahn SH, Kim BJ. Location of cerebral atherosclerosis: why is there a difference between east and west? Int J Stroke 2016 May 4. pii: 1747493016647736. [Epub ahead of print]

12. Kim JS, Nah HW, Park SM, Kim SK, Cho KH, Lee J, et al. Risk factors and stroke mechanisms in atherosclerotic stroke: intracranial compared with extracranial and anterior compared with posterior circulation disease. Stroke 2012;43:33133318.

13. Kim YD, Choi HY, Jung YH, Nam CM, Yang JH, Cho HJ, et al. Classic risk factors for atherosclerosis are not major determinants for location of extracranial or intracranial cerebral atherosclerosis. Neuroepidemiology 2009;32:201-207.

14. Lei $C$, Wu B, Liu M, Chen Y. Risk factors and clinical outcomes associated with intracranial and extracranial atherosclerotic stenosis acute ischemic stroke. J Stroke Cerebrovasc Dis 2014;23:1112-1117.

15. Resch JA, Okabe N, Loewenson R, Kimoto K, Katsuki S, Baker AB. A comparative study of cerebral atherosclerosis in a Japanese and Minnesota population. J Atheroscler Res 1967;7:687693.

16. McGarry P, Solberg LA, Guzman MA, Strong JP. Cerebral atherosclerosis in New Orleans. Comparisons of lesions by age, sex, and race. Lab Invest 1985;52:533-539. 
17. Nakamura $M$, Yamamoto $H$, Kikuchi $Y$, Ishihara $Y$, Sata T. Cerebral atherosclerosis in Japanese. I. Age related to atherosclerosis. Stroke 1971;2:400-408.

18. Solberg LA, McGarry PA, Moossy J, Tejada C, Loken AC, Robertson $W B$, et al. Distribution of cerebral atherosclerosis by geographic location, race, and sex. Lab Invest 1968;18:604-612.

19. D'Armiento FP, Bianchi A, de Nigris F, Capuzzi DM, D'Armiento MR, Crimi G, et al. Age-related effects on atherogenesis and scavenger enzymes of intracranial and extracranial arteries in men without classic risk factors for atherosclerosis. Stroke 2001;32:2472-2479.

20. Leung SY, Ng TH, Yuen ST, Lauder IJ, Ho FC. Pattern of cerebral atherosclerosis in Hong Kong Chinese. Severity in intracranial and extracranial vessels. Stroke 1993;24:779-786.

21. Sacco RL, Kargman DE, Gu Q, Zamanillo MC. Race-ethnicity and determinants of intracranial atherosclerotic cerebral infarction. The Northern Manhattan Stroke Study. Stroke 1995;26:14-20.

22. Leng $X$, Wong KS, Liebeskind DS. Evaluating intracranial atherosclerosis rather than intracranial stenosis. Stroke 2014:45:645-651.

23. Niccoli G, Liuzzo G, Montone RA, Crea F. Advances in mechanisms, imaging and management of the unstable plaque. Atherosclerosis 2014;233:467-477.

24. Nam HS, Han SW, Lee JY, Ahn SH, Ha JW, Rim SJ, et al. Association of aortic plaque with intracranial atherosclerosis in patients with stroke. Neurology 2006;67:1184-1188.

25. Kronzon I, Tunick PA. Aortic atherosclerotic disease and stroke. Circulation 2006;114:63-75.

26. Bentzon JF, Otsuka F, Virmani R, Falk E. Mechanisms of plaque formation and rupture. Circ Res 2014;114:1852-1866.

27. Bae HJ, Yoon BW, Kang DW, Koo JS, Lee SH, Kim KB, et al. Correlation of coronary and cerebral atherosclerosis: difference between extracranial and intracranial arteries. Cerebrovasc Dis 2006;21:112-119.

28. Ritz K, Denswil NP, Stam OC, van Lieshout JJ, Daemen MJ. Cause and mechanisms of intracranial atherosclerosis. Circulation 2014;130:1407-1414.

29. Ross R. Atherosclerosis--an inflammatory disease. N Engl J Med 1999;340:115-126.

30. Portanova A, Hakakian N, Mikulis DJ, Virmani R, Abdalla WM, Wasserman BA. Intracranial vasa vasorum: insights and implications for imaging. Radiology 2013;267:667-679.

31. Wolinsky H, Glagov S. Nature of species differences in the medial distribution of aortic vasa vasorum in mammals. Circ Res 1967;20:409-421.

32. Geiringer E. Intimal vascularization and atherosclerosis. J Pathol Bacteriol 1951;63:201-211.
33. Connolly ES Jr, Huang J, Goldman JE, Holtzman RN. Immunohistochemical detection of intracranial vasa vasorum: a human autopsy study. Neurosurgery 1996;38:789-793.

34. Aydin F. Do human intracranial arteries lack vasa vasorum? A comparative immunohistochemical study of intracranial and systemic arteries. Acta Neuropathol 1998;96:22-28.

35. Kwon TG, Lerman LO, Lerman A. The vasa vasorum in atherosclerosis: the vessel within the vascular wall. J Am Coll Cardiol 2015;65:2478-2480.

36. Finn AV, Jain RK. Coronary plaque neovascularization and hemorrhage: a potential target for plaque stabilization? JACC Cardiovasc Imaging 2010;3:41-44.

37. Maiellaro K, Taylor WR. The role of the adventitia in vascular inflammation. Cardiovasc Res 2007;75:640-648.

38. Ritman EL, Lerman A. The dynamic vasa vasorum. Cardiovasc Res 2007;75:649-658.

39. Dunmore BJ, McCarthy MJ, Naylor AR, Brindle NP. Carotid plaque instability and ischemic symptoms are linked to immaturity of microvessels within plaques. J Vasc Surg 2007:45:155-159.

40. Xu J, Lu X, Shi GP. Vasa vasorum in atherosclerosis and clinical significance. Int J Mol Sci 2015;16:11574-11608.

41. MacAlpin RN, Abbasi AS, Grollman JH Jr, Eber L. Human coronary artery size during life. A cinearteriographic study. $R a-$ diology 1973;108:567-576.

42. Gabrielsen TO, Greitz T. Normal size of the internal carotid, middle cerebral and anterior cerebral arteries. Acta Radiol Diagn (Stockh) 1970;10:1-10.

43. Funabashi N, Kobayashi $Y$, Perlroth M, Rubin GD. Coronary artery: quantitative evaluation of normal diameter determined with electron-beam CT compared with cine coronary angiography initial experience. Radiology 2003;226:263-271.

44. Krejza J, Arkuszewski M, Kasner SE, Weigele J, Ustymowicz A, Hurst RW, et al. Carotid artery diameter in men and women and the relation to body and neck size. Stroke 2006;37:11031105.

45. Gutierrez J, Rosoklija G, Murray J, Chon C, Elkind MS, Goldman J, et al. A quantitative perspective to the study of brain arterial remodeling of donors with and without HIV in the Brain Arterial Remodeling Study (BARS). Front Physiol 2014;5:56

46. Waller BF, Orr CM, Slack JD, Pinkerton CA, Van Tassel J, Peters T. Anatomy, histology, and pathology of coronary arteries: a review relevant to new interventional and imaging techniques--part I. Clin Cardiol 1992;15:451-457.

47. Pu Y, Dou $X$, Liu L. Natural history of intracranial atherosclerotic disease. Front Neurol 2014;5:125.

48. Finn AV, Nakano M, Narula J, Kolodgie FD, Virmani R. Con- 
cept of vulnerable/unstable plaque. Arterioscler Thromb Vasc Biol 2010;30:1282-1292.

49. Naghavi M, Libby P, Falk E, Casscells SW, Litovsky S, Rumberger J, et al. From vulnerable plaque to vulnerable patient: a call for new definitions and risk assessment strategies: part I. Circulation 2003;108:1664-1672.

50. Qureshi Al, Caplan LR. Intracranial atherosclerosis. Lancet 2014;383:984-998.

51. Velican D, Anghelescu M, Petrescu C, Velican C. Method dependent limits in a study on the natural history of coronary and cerebral atherosclerosis. Med Interne 1982;20:215-229.

52. Denswil NP, van der Wal AC, Ritz $K_{1}$ de Boer OJ, Aronica $E_{1}$ Troost $D$, et al. Atherosclerosis in the circle of willis: spatial differences in composition and in distribution of plaques. Atherosclerosis 2016;251:78-84.

53. Teng Z, Sadat U, Brown AJ, Gillard JH. Plaque hemorrhage in carotid artery disease: pathogenesis, clinical and biomechanical considerations. J Biomech 2014;47:847-858.

54. Derksen WJ, Peeters W, van Lammeren GW, Tersteeg C, de Vries JP, de Kleijn DP, et al. Different stages of intraplaque hemorrhage are associated with different plaque phenotypes: a large histopathological study in 794 carotid and 276 femoral endarterectomy specimens. Atherosclerosis 2011;218:369-377.

55. Harteveld AA, Denswil NP, Siero JC, Zwanenburg JJ, Vink A, Pouran $B$, et al. Quantitative intracranial atherosclerotic plaque characterization at 7T MRI: an ex vivo study with histologic validation. AJNR Am J Neuroradiol 2016;37:802-810.

56. Chen XY, Wong KS, Lam WW, Zhao HL, Ng HK. Middle cerebral artery atherosclerosis: histological comparison between plaques associated with and not associated with infarct in a postmortem study. Cerebrovasc Dis 2008;25:74-80.

57. Xu WH, Li ML, Gao S, Ni J, Yao M, Zhou LX, et al. Middle cerebral artery intraplaque hemorrhage: prevalence and clinical relevance. Ann Neurol 2012;71:195-198.

58. Chen XY, Lam WW, Ng HK, Fan YH, Wong KS. Intracranial artery calcification: a newly identified risk factor of ischemic stroke. J Neuroimaging 2007;17:300-303.

59. Wu XH, Chen XY, Wang $\sqcup$, Wong KS. Intracranial artery calcification and its clinical significance. J Clin Neurol 2016;12:253-261.

60. Wu XH, Chen XY, Fan YH, Leung TW, Wong KS. High extent of intracranial carotid artery calcification is associated with downstream microemboli in stroke patients. J Stroke Cerebrovasc Dis 2017;26:442-447.

61. Chen XY, Lam WW, Ng HK, Fan YH, Wong KS. The frequency and determinants of calcification in intracranial arteries in Chinese patients who underwent computed tomography ex- aminations. Cerebrovasc Dis 2006;21:91-97.

62. Virmani R, Ladich ER, Burke AP, Kolodgie FD. Histopathology of carotid atherosclerotic disease. Neurosurgery 2006;59(5 Suppl 3):S219-S227; discussion S3-S13.

63. Labadzhyan A, Csiba L, Narula N, Zhou J, Narula J, Fisher M. Histopathologic evaluation of basilar artery atherosclerosis. $J$ Neurol Sci 2011;307:97-99.

64. Stary HC, Chandler AB, Dinsmore RE, Fuster V, Glagov S, Insull $W \mathrm{Jr}$, et al. A definition of advanced types of atherosclerotic lesions and a histological classification of atherosclerosis. A report from the Committee on Vascular Lesions of the Council on Arteriosclerosis, American Heart Association. Circulation 1995;92:1355-1374.

65. Mazighi M, Labreuche J, Gongora-Rivera F, Duyckaerts C, Hauw JJ, Amarenco P. Autopsy prevalence of intracranial atherosclerosis in patients with fatal stroke. Stroke 2008; 39:1142-1147.

66. Gutierrez J, Elkind MS, Virmani R, Goldman J, Honig L, Morgello $\mathrm{S}$, et al. A pathological perspective on the natural history of cerebral atherosclerosis. Int J Stroke 2015;10:1074-1080.

67. Kasner SE, Chimowitz MI, Lynn MJ, Howlett-Smith H, Stern BJ, Hertzberg VS, et al. Predictors of ischemic stroke in the territory of a symptomatic intracranial arterial stenosis. Circulation 2006;113:555-563.

68. Wang Y, Zhao X, Liu L, Soo YO, Pu Y, Pan Y, et al. Prevalence and outcomes of symptomatic intracranial large artery stenoses and occlusions in China: the Chinese Intracranial Atherosclerosis (CICAS) Study. Stroke 2014;45:663-669.

69. Dieleman N, van der Kolk AG, Zwanenburg JJ, Harteveld AA, Biessels GJ, Luijten PR, et al. Imaging intracranial vessel wall pathology with magnetic resonance imaging: current prospects and future directions. Circulation 2014;130:192-201.

70. van der Kolk AG, Zwanenburg JJ, Brundel M, Biessels GJ, Visser $F$, Luijten $P R$, et al. Intracranial vessel wall imaging at 7.0-T MRI. Stroke 2011;42:2478-2484.

71. Kim YS, Lim SH, Oh KW, Kim JY, Koh SH, Kim J, et al. The advantage of high-resolution MRI in evaluating basilar plaques: a comparison study with MRA. Atherosclerosis 2012;224:411416.

72. Li ML, Xu WH, Song L, Feng F, You H, Ni J, et al. Atherosclerosis of middle cerebral artery: evaluation with high-resolution MR imaging at 3T. Atherosclerosis 2009;204:447-452.

73. Xu WH, Li ML, Gao S, Ni J, Zhou LX, Yao M, et al. Plaque distribution of stenotic middle cerebral artery and its clinical relevance. Stroke 2011;42:2957-2959.

74. Natori T, Sasaki M, Miyoshi M, Ohba H, Katsura N, Yamagu-

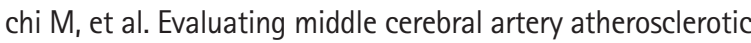
lesions in acute ischemic stroke using magnetic resonance 
T1-weighted 3-dimensional vessel wall imaging. J Stroke Cerebrovasc Dis 2014;23:706-711.

75. Choi YJ, Jung SC, Lee DH. Vessel wall imaging of the intracranial and cervical carotid arteries. J Stroke 2015;17:238255.

76. Yuan C, Mitsumori LM, Ferguson MS, Polissar NL, Echelard D, Ortiz G, et al. In vivo accuracy of multispectral magnetic resonance imaging for identifying lipid-rich necrotic cores and intraplaque hemorrhage in advanced human carotid plaques. Circulation 2001;104:2051-2056.

77. Narumi S, Sasaki M, Ohba H, Ogasawara K, Kobayashi M, Natori T, et al. Predicting carotid plaque characteristics using quantitative color-coded T1-weighted MR plaque imaging: correlation with carotid endarterectomy specimens. AJNR Am J Neuroradiol 2014;35:766-771.

78. Cai J, Hatsukami TS, Ferguson MS, Kerwin WS, Saam T, Chu $B$, et al. In vivo quantitative measurement of intact fibrous cap and lipid-rich necrotic core size in atherosclerotic carotid plaque: comparison of high-resolution, contrast-enhanced magnetic resonance imaging and histology. Circulation 2005;112:3437-3444.

79. den Hartog AG, Bovens SM, Koning W, Hendrikse J, Luijten PR, Moll $F L$, et al. Current status of clinical magnetic resonance imaging for plaque characterisation in patients with carotid artery stenosis. Eur J Vasc Endovasc Surg 2013;45:7-21.

80. Chen XY, Wong KS, Lam WW, Ng HK. High signal on T1 sequence of magnetic resonance imaging confirmed to be intraplaque haemorrhage by histology in middle cerebral artery. Int J Stroke 2014;9:E19.

81. Yang WJ, Chen XY, Zhao HL, Niu CB, Zhang B, Xu Y, et al. Postmortem study of validation of low signal on fat-suppressed T1-weighted magnetic resonance imaging as marker of lipid core in middle cerebral artery atherosclerosis. Stroke 2016;47:2299-2304.

82. Jiang $Y$, Zhu $C$, Peng $W$, Degnan $A J$, Chen $L$, Wang $X$, et al. Ex-vivo imaging and plaque type classification of intracranial atherosclerotic plaque using high resolution MRI. Atherosclerosis 2016;249:10-16.

83. Majidi S, Sein J, Watanabe M, Hassan AE, Van de Moortele $\mathrm{PF}$, Suri MF, et al. Intracranial-derived atherosclerosis assessment: an in vitro comparison between virtual histology by intravascular ultrasonography, 7T MRI, and histopathologic findings. AJNR Am J Neuroradiol 2013;34:2259-2264.

84. van der Kolk AG, Zwanenburg JJ, Denswil NP, Vink A, Spliet
WG, Daemen MJ, et al. Imaging the intracranial atherosclerotic vessel wall using 7T MRI: initial comparison with histopathology. AJNR Am J Neuroradiol 2015;36:694-701.

85. Mossa-Basha M, Hwang WD, De Havenon A, Hippe D, Balu $\mathrm{N}$, Becker $\mathrm{KJ}$, et al. Multicontrast high-resolution vessel wall magnetic resonance imaging and its value in differentiating intracranial vasculopathic processes. Stroke 2015;46:15671573.

86. Xu WH, Li ML, Gao S, Ni J, Zhou LX, Yao M, et al. In vivo highresolution MR imaging of symptomatic and asymptomatic middle cerebral artery atherosclerotic stenosis. Atherosclerosis 2010;212:507-511.

87. Millon A, Boussel L, Brevet M, Mathevet JL, Canet-Soulas $E$,

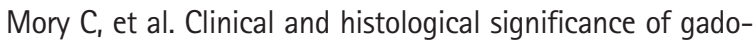
linium enhancement in carotid atherosclerotic plaque. Stroke 2012;43:3023-3028.

88. Wasserman BA. Advanced contrast-enhanced MRI for looking beyond the lumen to predict stroke: building a risk profile for carotid plaque. Stroke 2010;41(10 Suppl):S12-S16.

89. Kerwin WS, O'Brien KD, Ferguson MS, Polissar N, Hatsukami TS, Yuan C. Inflammation in carotid atherosclerotic plaque: a dynamic contrast-enhanced MR imaging study. Radiology 2006;241:459-468.

90. Ryu CW, Jahng GH, Shin HS. Gadolinium enhancement of atherosclerotic plaque in the middle cerebral artery: relation to symptoms and degree of stenosis. AJNR Am J Neuroradiol 2014;35:2306-2310.

91. Natori T, Sasaki M, Miyoshi M, Ito K, Ohba H, Miyazawa $H_{\text {, et }}$ al. Intracranial plaque characterization in patients with acute ischemic stroke using pre- and post-contrast three-dimensional magnetic resonance vessel wall imaging. J Stroke Cerebrovasc Dis 2016;25:1425-1430.

92. Qiao Y, Zeiler SR, Mirbagheri $S$, Leigh $R$, Urrutia V, Wityk $R$, et al. Intracranial plaque enhancement in patients with cerebrovascular events on high-spatial-resolution MR images. Radiology 2014;271:534-542.

93. Skarpathiotakis M, Mandell DM, Swartz RH, Tomlinson G, Mikulis DJ. Intracranial atherosclerotic plaque enhancement in patients with ischemic stroke. AJNR Am J Neuroradiol 2013;34:299-304.

94. Turan TN, Rumboldt Z, Granholm AC, Columbo L, Welsh CT, Lopes-Virella MF, et al. Intracranial atherosclerosis: correlation between in-vivo $3 \mathrm{~T}$ high resolution $\mathrm{MRI}$ and pathology. Atherosclerosis 2014;237:460-463. 
Supplementary Methods 1. Histology processing and analysis.

Bilateral MCA, VA, and BA were extracted for each of the 32 subjects and divided into 2-cm blocks, starting from the proximal and progressing to the distal segments. The small parts were dissected and decalcified in 1\% formic acid, followed by perfusion fixation in fresh 30\% formaldehyde. Serial sections of the isolated arteries were cut transversely at 4-mm intervals and embedded in paraffin. Sections were cut in $5 \mu \mathrm{m}$ thick and stained with hematoxylin-eosin (H\&E) and Victoria blue.

The histological sections were photographed by using a Leica DC 200 digital microscope (Leica Microsystems, Wetzler, Germany), and the slides with the most severe stenosis for each large artery were quantitatively measured using Image-Pro Plus software (Media Cybernetics, Silver Spring, MD, USA). One side of MCA and VA of the more affected by atherosclerosis were selected for each subject for the following morphological measurements. The internal elastic membrane in Victoria blue staining was traced and its length was recorded as "P". Arterial diameter was obtained by the formula: $D=P / \pi$. Original luminal area "A" was determined by the formula: $A=P / 4 \pi$. The area of atherosclerotic plaque was then traced and recorded as "Ai". The percentage of luminal stenosis was determined by the formula: (Ai/A) $\times 100 \%$. The medial and adventitial thickness were measured and recorded respectively. 
Supplementary Table 1. Clinical characteristics of 32 autopsy cases

\begin{tabular}{lc}
\hline & Total numbers \\
\hline Age & $71(45-97)$ \\
Male & $23(72)$ \\
Smoker & $9(28)$ \\
Hypertension & $9(28)$ \\
Diabetes & $6(19)$ \\
Ischemic heart disease & $9(28)$ \\
Ischemic stroke & $14(44)$ \\
Hemorrhagic stroke & $2(6)$ \\
\hline
\end{tabular}

Values are presented as median (interquartile range) or number (\%). 